\title{
A new preparation method for neutron monitor using ink-jet printer
}

\author{
By K. Takamiya ${ }^{1, *}$, M. Takimoto ${ }^{1}$, S. Shibata ${ }^{1}$, R. Okumura ${ }^{1}$, Y. Nakano ${ }^{1}$, J. H. Moon ${ }^{2}$ and S. H. Kim ${ }^{2}$ \\ ${ }^{1}$ Research Reactor Institute, Kyoto University, Kumatori-cho, Sennan-gun, Osaka, Japan \\ 2 Korea Atomic Energy Research Institute, Daeduk-daero 1045, Yuseong-gu, Daejeon, Korea
}

(Received December 22, 2009; accepted in revised form December 20, 2010)

\section{Neutron distribution / Neutron monitor / Ink-jet printer / Target preparation}

\begin{abstract}
Summary. A new method for preparing neutron monitors has been developed by using an ink-jet printer. A solution including gold, which is a monitor element for neutrons, is sprayed out uniformly onto a paper sheet in this method. Uniformity of the prepared monitor was found to be within a range of a few percent. The preparation procedure is simple and uses commercially available equipment without need for special modifications. Neutron distribution can be measured using a neutron monitor prepared by the method reported here.
\end{abstract}

\section{Introduction}

Monitoring neutron fluence and distribution is important in neutron irradiation experiments such as neutron activation analysis (NAA), prompt gamma-ray analysis, neutron radiography, etc. Various instruments and methods for neutron monitoring have been proposed to date including fission chamber, scintillation detector, imaging plate, activation analysis, etc. An imaging plate method [1] is one of the most convenient methods for monitoring neutron fluence and distribution. This is especially true in experiments using neutron beams, such as neutron radiography and prompt gamma-ray analysis. In the case of monitoring neutrons in small spaces such as research reactor irradiation holes, activation analysis methods are the most convenient because placing and retrieving monitor materials is easy [2]. Metallic wires or foils are often used as monitor materials in an activation analysis method $[3,4]$. Monitor materials are often transported to neutron irradiation holes by pneumatic transport tubes $[1,5]$. After neutron irradiation at the irradiation hole, activated monitor materials are sent back to a laboratory room. Neutron fluence can be estimated from activities of the irradiated monitor materials. But, the self-absorption effect causes unequal activation in neutron irradiation when using thick metallic wire as a monitor material. In addition, the self-shielding effect causes incorrect analysis of gammaray spectra. In the case of using metallic foils, those effects are negligible because of the thinness of the foil, but difficulty in handling thin foils may induce experimental errors.

*Author for correspondence

(E-mail: takamiya@ rri.kyoto-u.ac.jp)
In this work, a new preparation method for a neutron monitor, using an ink-jet printer, has been developed. Metal solution is applied on a paper sheet using an ink-jet printer to prepare neutron monitor material. The prepared monitor is thin enough to avoid self-absorption and self-shielding effects, and is easy to handle compared with metallic foil. The amount of metal applied and the size of monitor are variable using software which controls the ink-jet printer. Neutron distribution at irradiation holes, guide tubes, and at other neutron irradiation facilities, can be observed using the developed neutron monitor.

\section{Experiments}

A commercial ink-jet printer and metal solution are used in the preparation procedure for the neutron monitor developed in this research. Ink mixed with a solution in which a metal irradiation target element was dissolved is applied on a paper sheet using the ink-jet printer to prepare the neutron monitor. Gold was used as the irradiation target in this research because gold is one of the most convenient elements for neutron fluence monitoring.

Two kinds of experiments were performed to investigate the characteristics of the developed neutron monitor: uniformity, and controllability of the amount of a target element on the monitor surface, by NAA method. Measurement of the neutron fluence distribution using the developed monitor was performed at a research reactor neutron irradiation facility to confirm the performance. Details of the prepared neutron monitors and conditions of neutron irradiation for each monitor are listed in Table 1.

\subsection{Preparation method of the neutron monitor}

A gold standard solution, $2 \mathrm{~mL}$ of $1000 \mathrm{mg} \mathrm{Au} / \mathrm{L}$ solution, and $2 \mathrm{~mL}$ of a yellow ink (BCI-7EY, Canon Co., Ltd.) were mixed. The mixed solution was poured into an empty ink cartridge, and the cartridge was attached to the ink-jet printer (PIXUS iP3500, Canon Co., Ltd.). The mixed solution in the cartridge was sprayed out onto paper under control of a PC as is usual for document printing. The printer software that can spray out four individual kinds of ink with desired concentrations was developed by VisualBasic.net. The amount of ink that is sprayed out is expressed as a percentage of the maximum concentration, which is listed in Table 1 as "concentration parameter". 
Table 1. List of preparation and irradiation conditions of neutron monitors.

\begin{tabular}{|c|c|c|c|}
\hline Sample & $\begin{array}{l}\text { Concentration } \\
\text { parameter }\end{array}$ & Size & $\begin{array}{l}\text { Irradiation } \\
\text { time }\end{array}$ \\
\hline $\begin{array}{l}\text { U1 } \\
\text { U2 } \\
\text { U3 } \\
\text { U4 } \\
\text { U5 }\end{array}$ & $100 \%$ & $10 \mathrm{~mm} \times 10 \mathrm{~mm}$ & $10 \mathrm{~min}$ \\
\hline $\begin{array}{l}1 \% \\
5 \% \\
10 \% \\
30 \% \\
60 \% \\
100 \%\end{array}$ & $\begin{array}{c}1 \% \\
5 \% \\
10 \% \\
30 \% \\
60 \% \\
100 \%\end{array}$ & $10 \mathrm{~mm} \times 10 \mathrm{~mm}$ & $10 \mathrm{~min}$ \\
\hline $\begin{array}{l}\text { For neutron } \\
\text { distribution }\end{array}$ & $100 \%$ & $\begin{array}{c}45 \mathrm{~mm} \times 60 \mathrm{~mm} \\
\text { (separated to nine } \\
5 \mathrm{~mm} \times 60 \mathrm{~mm} \text { pieces })\end{array}$ & $10 \mathrm{~min}$ \\
\hline
\end{tabular}

The four kinds of inks (cyan, magenta, yellow, and black) and the paper used in this work were analyzed by NAA before the gold printing experiments to determine the comprised impurity. It was found that there was no interfering element in the yellow ink or the paper for measurements of gamma-rays from ${ }^{198} \mathrm{Au}$, which is produced by neutron irradiation of gold. And it was decided to adopt yellow ink as a solvent for the gold solution.

\subsection{Tests for uniformity and controllability}

Five monitors, named as U1-5, were prepared under the same conditions and the amount of gold in each monitor was determined by NAA at the research reactor HANARO (High-flux Advanced Neutron Application ReactOr), in the Korea Atomic Energy Research Institute, to investigate the uniformity of the prepared monitors. Five squares $(10 \mathrm{~mm} \times 10 \mathrm{~mm})$ were printed by the ink-jet printer at one time with the maximum gold concentration on discrete areas of a paper sheet. Square pieces were detached from the paper sheet to be the monitors and encapsulated in polyethylene bags separately. A standard sample containing $20 \mu \mathrm{g}$ of gold, which was prepared by drying up drops of gold standard solution $(1,000 \mathrm{mg} \mathrm{Au} / \mathrm{L})$ on filter paper, was also encapsulated in another polyethylene bag. All the polyethylene bags were packed in an envelope together and encapsulated in an irradiation rabbit. The rabbit containing the monitors was irradiated by neutrons using PTS\#1 (Pneumatic Transfer System \#1) at HANARO. Because the uniformity of neutron fluence in PTS\#1 was confirmed by the previous experiment [2], all samples were irradiated by neutrons equally in this irradiation. After the irradiation time of $10 \mathrm{~min}$, gamma-ray measurements were carried out on all irradiated samples using a Ge-detector (HP-Ge detector, GEM 35185P, ORTEC, USA).

For the test of the controllability of the amount of target element, six squares $(10 \mathrm{~mm} \times 10 \mathrm{~mm})$ were printed with different concentration parameters $(1,5,10,30,60$ and $100 \%$ of the maximum gold concentration), as listed in Table 1. Each square piece was detached from the printing sheet and encapsulated in a polyethylene bag. Neutron irradiation was carried out using PTS\#1 twice separately. The irradiation times were $30 \mathrm{~min}$ for $1-10 \%$ samples and $10 \mathrm{~min}$ for $30-100 \%$ ones. For each irradiation, a standard sample containing $20 \mu \mathrm{g}$ of gold was prepared and irradiated together. Gamma-ray spectra were measured for the irradiated samples by the Ge-detector after the neutron irradiations.

\subsection{Measurement of neutron distribution}

A measurement of axial neutron distribution at the position of the irradiation rabbit in the PTS\#1 of HANARO was undertaken using the developed neutron monitor. As a neutron monitor, a paper piece measuring $45 \mathrm{~mm} \times 60 \mathrm{~mm}$ was prepared with the maximum gold concentration. The paper piece was rolled up and inserted into the rabbit so that the short side of the paper piece was along the vertical direction of the rabbit. After neutron irradiation of $10 \mathrm{~min}$ using PTS\#1, the neutron monitor was separated into nine $5 \mathrm{~mm} \times 60 \mathrm{~mm}$ pieces. The neutron monitor and irradiation setup are shown in Fig. 1. Measurements of gamma-ray spectra for the separated pieces were carried out using the Ge-detector.

Spectral analysis was performed using FitzPeaks software (JF Computing Services, Stanford-in-the-Vale, UK) for all spectra obtained in this work. The amount of gold contained in each neutron monitor was determined from the calculated photo-peak area for the monitors and the irradiated gold standard.

\section{Results and discussion}

Fig. 2 shows the estimated weights of gold contained in the neutron monitors of the $10 \mathrm{~mm} \times 10 \mathrm{~mm}$ samples with $100 \%$ gold concentration. Error bars in the figure represent one standard deviation, which are estimated on the basis of statistical error and fitting error in gamma-spectrum analysis. All the determined weights of gold correspond to each other within their error ranges. The uniformity of the monitor developed in this method is confirmed from this result. The averaged weight of gold contained in the $100 \mathrm{~mm}^{2}$ monitors is $5.6 \pm 0.1 \mathrm{ng}$. This amount is comparable to gold foil with a thickness of about $30 \mathrm{~nm}$, which means the selfabsorption effect is negligible in neutron irradiation of the monitor.

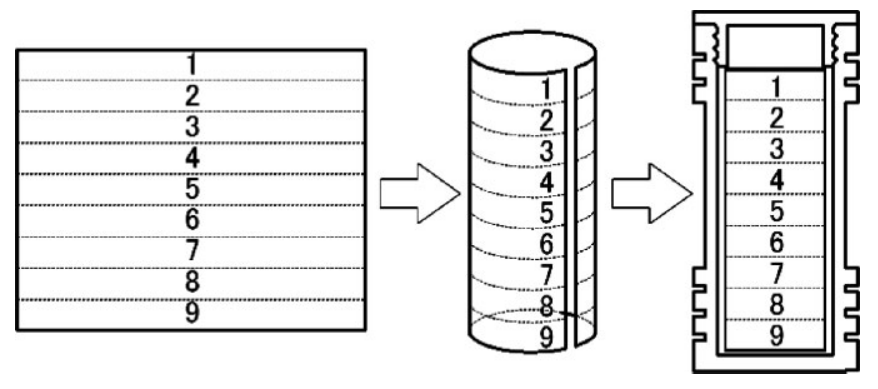

Fig. 1. The setup of the neutron monitor for the neutron distribution measurement. The right-side drawing shows a cross section of the irradiation rabbit in which the neutron monitor is inserted. 


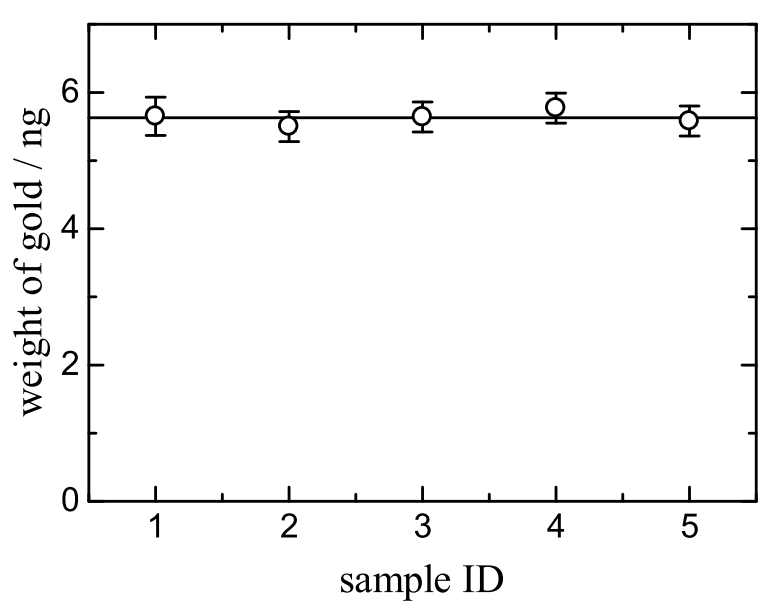

Fig. 2. Variation of weights of gold contained in five neutron monitors prepared under the same conditions.

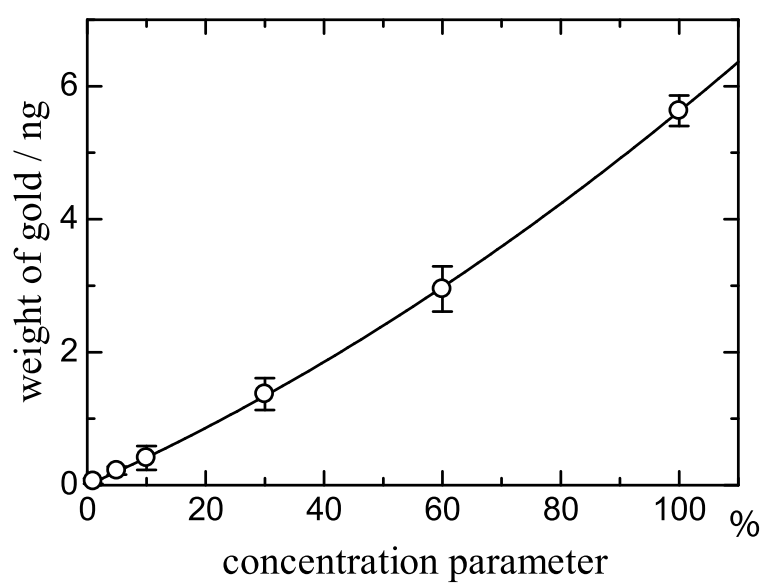

Fig. 3. The correlation between determined weights of gold and concentration parameters $(\%)$.

Fig. 3 shows the determined weights of gold contained in the monitor as a function of the concentration parameters input to the printing software. Error bars in the figure represent one standard deviation. A linear correlation between the gold weight and the concentration parameter was expected, but the result does not show a linear correlation. In this figure, the solid line shows the result fitted by a quadratic curve. This nonlinear correlation was caused by a "gamma correction" performed by the printing software of the ink-jet printer. As just described, there is not a linear correlation between the gold weight and the concentration parameter, but it was found that the amount of applied gold can be controlled by using the obtained correlation for calibration parameters.

The developed neutron monitor was used to measure the axial neutron distribution of the irradiation hole in HANARO. The neutron distribution observed in this research is the distribution of the neutrons in an energy range which can induce the neutron capture reaction of ${ }^{197} \mathrm{Au}$. The activity of ${ }^{198} \mathrm{Au}$ which was produced in each of nine monitor pieces separated from the original $45 \mathrm{~mm} \times 60 \mathrm{~mm}$ neutron monitor (Fig. 1) is shown in Fig. 4. In this figure, the vertical axis shows relative activity of ${ }^{198} \mathrm{Au}$ produced in each monitor piece in relation to the averaged activity of all the pieces, and the numbers indicated below the horizontal axis show the

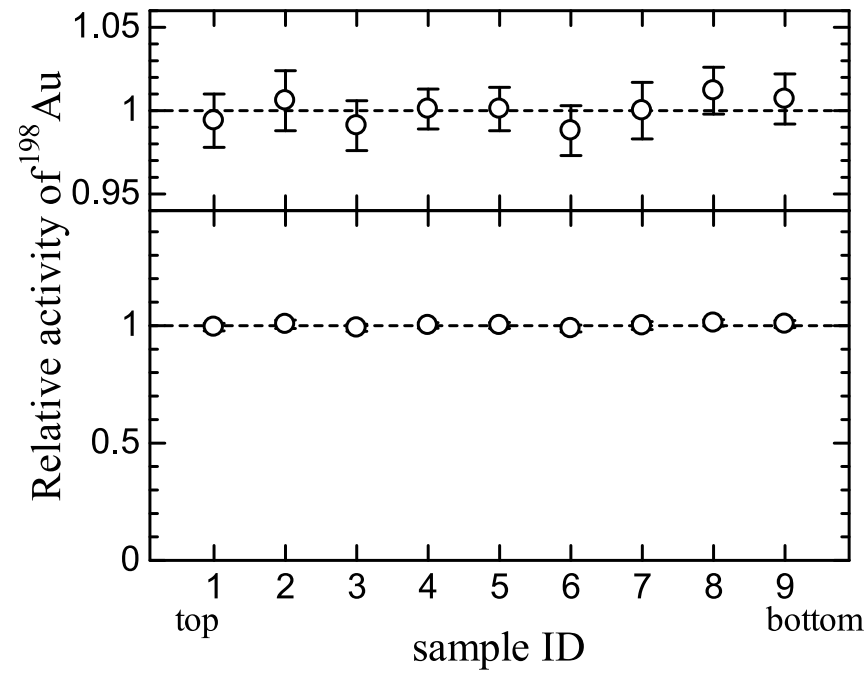

Fig. 4. The relative activity of ${ }^{198} \mathrm{Au}$ produced in monitor pieces separated from the neutron monitor. The upper panel shows the same data in a narrower range around the average value.

sample IDs. The expanded figure for the relative activity of ${ }^{198} \mathrm{Au}$ in the range of 0.95 to 1.05 is shown in the upper panel of this figure. Error bars in the figure represent one standard deviation. The activities of nine pieces correspond to each other within their error ranges. A trend or fluctuation of neutron fluence was not observed using the developed neutron monitor. This result is consistent with the result of a neutron flux measurement previously performed using $\mathrm{Au}-\mathrm{Al}$ wire monitors [2].

\section{Conclusion}

A new technique for preparing neutron monitors using an ink-jet printer was developed in the present work. The uniformity of the monitors was investigated and found to be within a range of a few percent. In the present preparation conditions there is no linear correlation between the applied weight of monitor material and the concentration parameter; however, the amount of monitor material is controllable using the obtained correlation curve between the weight and the concentration parameter. The neutron distribution measurement was conducted using the neutron monitor developed in this research. We found that the developed method for preparation of neutron monitors is convenient and useful. In the future, we will try to measure neutron distributions using an imaging plate combined with the developed monitors in order to more closely and more easily observe neutron distributions.

Acknowledgment. The authors would like to thank the staff of HANARO, Korean Atomic Energy Research Institute for their help on neutron irradiation experiments. This work was supported by JSPS KAKENHI 21760707.

\section{References}

1. Tazaki, S., Neriishi, K., Takahashi, K., Etoh, M., Karasawa, Y., Kumazawa, S., Niimura, N.: Development of a new type of imaging plate for neutron detection. Nucl. Instrum. Methods A 424, 20-25 (1999).

2. Chung, Y. S., Kim, S. H., Moon, J. H., Baek, S. Y., Kim, Y. J., Kim, H. R.: New pneumatic transfer systems for neutron activa- 
tion analysis at the HANARO research reactor. J. Radioanal. Nucl. Chem. 278, 707-712 (2008).

3. Khattab, K., Ghazi, N., Omar, H.: Determination of the axial thermal neutron flux non-uniform factor in the MNSR inner irradiation capsule. Nucl. Instrum. Methods A 575, 456-460 (2007).

4. Fujibuchi, T., Yamaguchi, I., Kasahara, T., Iimori, T., Masuda, Y., Kimura, K., Watanabe, H., Isobe, T., Sakae, T.: Measurement of thermal neutron fluence distribution with use of ${ }^{23} \mathrm{Na}$ radioactivation around a medical compact cyclotron. Radiol. Phys. Technol. 2, 159-165 (2009)

5. Takamiya, K., Okumura, R., Abe, N., Nakano, Y., Miyata, K., Fukutani, S., Taniguchi, A., Yamana, H.: Development of a new control system for pneumatic transportation facility in KUR. J. Radioanal. Nucl. Chem. 278, 719-721 (2008). 